\title{
UNIVALENCE CRITERIA IN MULTIPLY-CONNECTED DOMAINS
}

\author{
BY
}

BRAD G. OSGOOD

\author{
Dedicated to the memory of Zeev Nehari
}

\begin{abstract}
Theorems due to Nehari and Ahlfors give sufficient conditions for the univalence of an analytic function in relation to the growth of its Schwarzian derivative. Nehari's theorem is for the unit disc and was generalized by Ahlfors to any simply-connected domain bounded by a quasiconformal circle. In both cases the growth is measured in terms of the hyperbolic metric of the domain. In this paper we prove a corresponding theorem for finitely-connected domains bounded by points and quasiconformal circles. Metrics other than the hyperbolic metric are also considered and similar results are obtained.
\end{abstract}

1. Introduction. In $1949 \mathrm{Z}$. Nehari [13] established a close connection between the univalence of a function $f$, analytic in the unit disc $B$, and the growth of its Schwarzian derivative,

$$
S_{f}=\left(\frac{f^{\prime \prime}}{f^{\prime}}\right)^{\prime}-\frac{1}{2}\left(\frac{f^{\prime \prime}}{f^{\prime}}\right)^{2} .
$$

THEOREM 1. In order that the function $f$, analytic in $B$, be univalent in $B$ it is necessary that

$$
\left|S_{f}(z)\right| \leqslant 6 /\left(1-|z|^{2}\right)^{2}, \quad z \in B,
$$

and sufficient that

$$
\left|S_{f}(z)\right| \leqslant 2 /\left(1-|z|^{2}\right)^{2}, \quad z \in B .
$$

The necessary condition seems to have first appeared in [9] and is shown to be sharp by taking $f$ to be the Koebe function. E. Hille [8] observed that 2 is the best possible constant in (3). Much later L. Ahlfors and G. Weill [2] proved that if the number 2 is replaced by $2 k, k<1$, in (3) then the function has a quasiconformal extension to the entire plane. Then in 1963 Ahlfors [1] obtained a significant generalization of these results to a broad class of simply-connected domains. Before stating this theorem it is necessary to recall several definitions.

A Jordan curve in the extended complex plane $\overline{\mathbf{C}}=\mathbf{C} \cup\{\infty\}$ is said to be a $K$-quasiconformal circle if it is the image of the unit circle under a $K$-quasiconformal mapping of $\overline{\mathbf{C}}$. The curve is said to be quasiconformal if it is $K$-quasiconformal

Received by the editors March 5, 1979. The principal results in this paper were announced at the Colloquium on Complex Analysis held in Joensuu, Finland, August 1978.

AMS (MOS) subject classifications (1970). Primary 30A36, 30A60; Secondary 30A24, 30A21.

Key words and phrases. Schwarzian derivative, hyperbolic metric, quasiconformal circle, kernel function. 
for some finite $K$ and the number

$$
K(C)=\inf \{K: f: \overline{\mathbf{C}} \rightarrow \overline{\mathbf{C}} \text { is } K \text {-quasiconformal and } f(\partial B)=C\}
$$

is often called the dilatation of $C$. Analytic Jordan curves are quasiconformal circles, but only circles and lines are 1-quasiconformal.

Next, if $D$ is a simply-connected domain in $\overline{\mathbf{C}}$ with at least three boundary points and $g$ is any conformal mapping of $D$ onto $B$ then the function

$$
\rho_{D}(z)=\left|g^{\prime}(z)\right| /\left(1-|g(z)|^{2}\right), \quad z \in D,
$$

is independent of the choice of conformal mapping and defines the density of the hyperbolic metric in $D$. If $\varphi$ is analytic in $D$ we define its hyperbolic norm to be

$$
\|\varphi\|_{D}=\sup _{z \in D}|\varphi(z)| \rho_{D}(z)^{-2}
$$

Ahlfors' theorem can now be stated.

THEOREM 2. If $\partial D$ is a $K$-quasiconformal circle then there is a positive constant $a$, depending only on $K$, such that $f$ is univalent in $D$ and has a quasiconformal extension to $\overline{\mathbf{C}}$ whenever $f$ is analytic in $D$ with $\left\|S_{f}\right\|_{D} \leqslant a$.

Note that if $\left\|S_{f}\right\|_{D}$ is bounded then $f$ is locally univalent in $D$ and hence $S_{f}$ as defined in (1) has an analytic extension to any possible poles of $f$ and to $\infty$ if $\infty \in D$.

If $D$ is an arbitrary domain in $\overline{\mathbf{C}}$ of hyperbolic type then we can take the unit disc to be its universal covering surface. If $p: B \rightarrow D$ is any analytic projection then the density of the hyperbolic metric in $D$ is defined by

$$
\rho_{D}(p(z))\left|p^{\prime}(z)\right|=\rho_{B}(z), \quad z \in D .
$$

Once again this is independent of the choice of $p$. The hyperbolic norm of an analytic function in $D$ is defined just as in (4).

Our main result is as follows.

THEOREM 3. Suppose that $D$ is a finitely-connected domain and that each component of $\partial D$ is either a point or a quasiconformal circle. Then there is a positive constant a, depending only on $D$, such that $f$ is univalent in $D$ whenever $f$ is analytic in $D$ with $\left\|S_{f}\right\|_{D} \leqslant a$.

The proof of Theorem 3 proceeds by decomposing $D$ into simply-connected subdomains in a way that makes it possible to apply Theorem 2 . Such decompositions are discussed in $\$ 2$ where a proof of Theorem 3 will be given. The proof depends on a lemma which will be proved in $\$ 3$. In $\$ 4$ we discuss some related results concerning metrics other than the hyperbolic metric and also consider the question of quasiconformal extension. $\$ 5$ is devoted to constructing an example of an infinitely-connected domain for which the conclusion of Theorem 3 is valid.

2. Quasiconformally decomposable domains. In order to apply Theorem 2 to prove Theorem 3 we shall show that any two points in a domain $D$ satisfying the hypotheses Theorem 3 can be placed in a simply-connected subdomain of $D$ 
bounded by a quasiconformal circle. To this end we introduce the following definition.

We say that a collection $\mathscr{D}$ of domains $\Delta \subset D$ is a $K$-quasiconformal decomposition of $D$ if each $\Delta \in \mathscr{D}$ is bounded by a $K$-quasiconformal circle and if any two points $z_{1}, z_{2} \in D$ lie in the closure of some $\Delta \in \mathscr{D}$. We shall refer to this second condition as the covering property of $\mathscr{D}$. We say that $D$ is quasiconformally decomposable if it has a $K$-quasiconformal decomposition for some finite $K$. A domain may have many different quasiconformal decompositions with different constants $K$ and the minimum such constant will depend on the domain.

Certainly any simply-connected domain bounded by a $K$-quasiconformal circle is $K$-quasiconformally decomposable. A more general converse also holds and will be proved in $\$ 4$.

The following lemma is an immediate consequence of the definition.

LEMMA 1. If $D$ has a $K_{1}$-quasiconformal decomposition $\mathscr{D}$ and if $f$ is a $K_{2}$-quasiconformal mapping of $\overline{\mathbf{C}}$ then $f(D)$ has a $K_{1} K_{2}$-quasiconformal decomposition $\mathscr{D}^{\prime}=$ $\{f(\Delta): \Delta \in \mathscr{D}\}$.

We now state the key lemma used for the proof of Theorem 3.

LeMMA 2. If $D$ is a finitely-connected domain and each component of $\partial D$ is either a point or a quasiconformal circle, then $D$ is quasiconformally decomposable.

The proof of this lemma will be given in $\$ 3$ where an explicit decomposition will be constructed. Lemma 1 will be used to reduce the problem to constructing a decomposition of a circle domain.

Assuming Lemma 2, the only other fact we require to prove Theorem 3 is a monotonicity property of the hyperbolic norm.

If $D^{\prime} \subset D$ and $\varphi$ is analytic in $D$ then

$$
\|\varphi\|_{D^{\prime}} \leqslant\|\varphi\|_{D}
$$

This follows from the well-known property of the hyperbolic density; if $D^{\prime} \subset D$ then $\rho_{D} \leqslant \rho_{D^{\prime}}$

Proof of Theorem 3. By Lemma $2 D$ is quasiconformally decomposable and we suppose that $\mathscr{D}$ is a $K$-quasiconformal decomposition where $K<\infty$ depends on $D$. Let $z_{1}, z_{2}$ be any two distinct points in $D$. Then there is a $\Delta \in \mathscr{D}$ with $z_{1}, z_{2} \in \bar{\Delta}$. Now by Theorem 2 there is a positive constant $a=a(K)$ such that $\left\|S_{f}\right\|_{\Delta} \leqslant a$ implies that $f \mid \Delta$ is univalent and has a quasiconformal extension to $\overline{\mathbf{C}}$. In particular we see that $f$ is actually univalent in $\bar{\Delta} \cap D$ and this covers the situation when either or both of $z_{1}, z_{2}$ lie on $\partial \Delta$.

Therefore if we choose $a$ in this way and suppose that $\left\|S_{f}\right\|_{D} \leqslant a$ then from (5) it follows that $f\left(z_{1}\right) \neq f\left(z_{2}\right)$ and hence $f$ is univalent in $D$.

It is possible to consider other metrics on $D$ for which the associated norm is monotonic with respect to the domain and for which Theorem 2 holds. Thus the property of quasiconformal decomposability and the above argument will yield univalence criteria in these cases as well. Such alternate formulations of Theorem 3 
will be considered in $\$ 4$. Also in $\$ 4$ we shall show how the constant $a$ can be chosen so as to imply that $f$ has a quasiconformal extension to $\overline{\mathbf{C}}$.

3. Proof of Lemma 2. The proof will be given in several stages, but the basic idea is this. By joining the boundary components of $D$ in a specified manner we can write $D$ as the union of simply-connected subdomains bounded by quasiconformal circles. The required decomposition will then be obtained by taking various unions of these subdomains. Ultimately we shall reduce the construction to the case where the joining is done by piecewise analytic arcs. We therefore begin this section with the following lemma.

LEMMA 3. If $C$ is a Jordan curve consisting of finitely many analytic arcs meeting at pairwise nonzero angles, then $C$ is a quasiconformal circle.

We omit the proof; see [12, pp. 97-105].

As a first step in the proof of Lemma 2 we show that isolated points are, in a sense, removable for quasiconformally decomposable domains. This will allow us to concentrate only on the case of nondegenerate boundary components.

LEMMA 4. If $D$ is a $K$-quasiconformally decomposable domain and if $E$ is $a$ set of $n$ points in $D$, then $D \backslash E$ is $K_{1}$-quasiconformally decomposable where $K_{n}$ depends only on $K$ and $n$.

Proof. We prove this by induction on $n$. First suppose that $E$ consists of the single point $\zeta$ and set $D^{\prime}=D \backslash\{\zeta\}$. Let $\mathscr{D}$ be a $K$-quasiconformal decomposition of $D$. We construct a quasiconformal decomposition $\mathscr{D}^{\prime}$ of $D^{\prime}$ from the sets in $\mathscr{D}$ in the following manner.

By definition there is at least one $\Delta \in \mathscr{D}$ with $\zeta \in \bar{\Delta}$. Define

$$
\mathscr{D}_{0}=\{\Delta \in \mathscr{D}: \zeta \in \partial \Delta\} \text {. }
$$

If $\mathscr{D}_{0}=\mathscr{D}$ then we can take $\mathscr{D}^{\prime}=\mathscr{D}=\mathscr{D}_{0}$, and hence $D^{\prime}$ is also $K$-quasiconformally decomposable.

If this is not the case then there is a $\Delta \in \mathscr{D}$ with $\zeta \in \Delta$. Since $\partial \Delta$ is a $K$-quasiconformal circle there is a $K$-quasiconformal mapping $g$ of $\overline{\mathbf{C}}$ such that $g(\partial B)=\partial \Delta$, and we may further assume that $g(B)=\Delta$ and $g(0)=\zeta$. We form three subdomains of $B^{\prime}=B \backslash\{0\}$;

$$
B_{1}=\left\{z \in B^{\prime}: 0<\arg (z)<4 \pi / 3\right\}, \quad B_{2}=e^{(2 \pi i / 3)} B_{1}, \quad B_{3}=e^{(4 \pi i / 3)} B_{1} .
$$

Thus each $B_{j}$ is two thirds of the punctured disc.

It is easy to see that $\left\{B_{1}, B_{2}, B_{3}\right\}$ is a quasiconformal decomposition of $B^{\prime}$. Indeed if $z_{1}, z_{2} \in B^{\prime}$ then they are at most diametrically opposite and so both $z_{1}$ and $z_{2}$ lie in the closure of at least one of the $B_{j}$. Furthermore by Lemma $3, \partial B_{1}$ is a quasiconformal circle. We shall show in $\S 5$ that actually $K\left(\partial B_{1}\right)<8 / 3$.

Now using Lemma 1 we see that the set $\left\{g\left(B_{j}\right): j=1,2,3\right\}$ is a $K_{1}$-quasiconformal decomposition, $\mathscr{D}_{\Delta}$, of $\Delta \backslash\{\zeta\}$, where $K_{1}=\frac{8}{3} K$. For each $\Delta$ in the set

$$
\mathscr{D}_{1}=\{\Delta \in \mathscr{D}: \zeta \in \Delta\}
$$


we can construct the same type $K_{1}$-quasiconformal decomposition $\mathscr{D}_{\Delta}$. Thus if we now define

$$
\mathscr{D}^{\prime}=\{\Delta \in \mathscr{D}: \zeta \notin \Delta\} \cup \underset{\Delta \in \mathscr{D}_{1}}{\bigcup} \mathscr{D}_{\Delta}
$$

then $\mathscr{D}^{\prime}$ is a $K_{1}$-quasiconformal decomposition of $D^{\prime}$.

From here the induction proceeds easily. Suppose that the theorem holds for $E$ of cardinality $n-1$ and consider $D \backslash\left\{\zeta_{1}, \ldots, \zeta_{n}\right\}$. By the induction hypothesis there is a $K_{n-1}$-quasiconformal decomposition of $D \backslash\left\{\zeta_{1}, \ldots, \zeta_{n-1}\right\}$. Using the above construction we obtain an $\frac{8}{3} K_{n-1}$-quasiconformal decomposition of $\left(D \backslash\left\{\zeta_{1}, \ldots, \zeta_{n-1}\right\}\right) \backslash\left\{\zeta_{n}\right\}$. This completes the proof with $K_{n}=\left(\frac{8}{3}\right)^{n} K$.

We now construct a quasiconformal decomposition of $D$ when $\partial D$ consists of a finite number of quasiconformal circles. By the preceding lemma this will be sufficient to include the case when $\partial D$ also contains a finite number of point components. In order to reduce the problem to a simpler situation we employ a result due to G. Springer [14].

Lemma 5. Suppose that $D$ and $D^{\prime}$ are two domains of the same finite connectivity and that the boundary components of each are quasiconformal circles. Then any quasiconformal mapping of $D$ onto $D^{\prime}$ extends to a quasiconformal mapping of $\overline{\mathbf{C}}$.

If we now map $D$ conformally onto a circle domain $D^{\prime}$, i.e., a domain all of whose boundary components are circles in the finite plane, then by Lemmas 1 and 5 it is sufficient to construct a quasiconformal decomposition of $D^{\prime}$. We therefore suppose that $D$ itself is a finitely-connected circle domain.

First consider the case when $D$ is a circular ring domain, which we may further assume to be the annulus

$$
D=\{z: 1<|z|<R\}
$$

The construction of the quasiconformal decomposition of $D$ is virtually the same as that of $B \backslash\{0\}$ in the proof of Lemma 4. We define

$$
D_{1}=\{z \in D: 0<\arg (z)<4 \pi / 3\}, \quad D_{2}=e^{(2 \pi i / 3)} D_{1}, \quad D_{3}=e^{(4 \pi i / 3)} D_{1} .
$$

From Lemma 3 we have that $\partial D_{1}$ is a quasiconformal circle and if $z_{1}, z_{2} \in D$ then both points lie in the closure of at least one of the $D_{j}$ just as before.

Now let $D$ be a circle domain with boundary circles $C_{0}, \ldots, C_{n-1}, n \geqslant 3$. To construct a quasiconformal decomposition in this final case we map $D$ conformally onto another canonical domain. Let $w=\Psi(z)=\Psi(z ; u, v)$ be a conformal mapping of $D$ onto the domain $D^{\prime}$ consisting of the entire plane minus $n$ rectilinear slits lying on rays from the origin. The radial slit mapping $\Psi$ has $\Psi(u)=0, \Psi(v)=\infty$ and is determined up to a multiplicative constant. Regarding boundary behavior, $\Psi$ has an analytic extension to $\bar{D}$ and the two points on $C_{j}$ that correspond to the endpoints of $\Psi\left(C_{j}\right)$ divide $C_{j}$ into two arcs each of which is mapped in a one-to-one fashion onto the slit. Thus we see that the preimage of a line segment meeting a slit $\Psi\left(C_{j}\right)$ will be an analytic arc forming a nonzero angle where it meets the circle $C_{j}$. This is certainly clear at the interior points of the slit and at the endpoints we shall 
only be concerned with a segment lying on the ray containing $\Psi\left(C_{j}\right)$ where again the conclusion is immediate.

The idea is to join the slits cyclically and then join the endpoints farthest from the origin to $\infty$ along the rays. This will partition $D^{\prime}$ into simply-connected subdomains, the preimages of which will form part of the quasiconformal decomposition of $D$. To carry this out we require the following lemma.

Lemma 6. For each $v \in D$ it is possible to choose $u \in D$ so that no two slits lie on the same ray in the image of $D$ under $\Psi(z ; u, v)$.

Proof. We shall actually show slightly more, namely that the set of points $u \in D$ for which the assertion is false is nowhere dense in $D$. This will follow from several important identities in [5]. We use the same notation appearing there and indicate only what is needed in our situation.

First let $\Phi(z ; u, v)$ be a conformal mapping of $D$ onto the entire plane minus $n$ concentric circular slits. Again $\Phi(u)=0, \Phi(v)=\infty$ and the mapping is determined up to a multiplicative constant. The boundary behavior of $\Phi$ is analogous to that of $\Psi$. Next, for $z \in C_{j}, j=0, \ldots, n-1$, define

$$
\begin{aligned}
\log \Phi(z ; u, v) & =\kappa_{j}(u, v)+i r_{j}(z ; u, v), \\
\log \Psi(z ; u, v) & =s_{j}(z ; u, v)+i \lambda_{j}(u, v), \\
k_{j}(u, v) & =\kappa_{j}(u, v)+i \lambda_{j}(u, v) .
\end{aligned}
$$

Under suitable normalizations of $\Phi$ and $\Psi$ it is shown in [5] that for each $j, k_{j}(u, v)$ is an analytic function of both $u$ and $v$. Furthermore, for each $v \in D$ the function

$$
R_{i j}(u)=\exp \left(k_{i}(u, v)-k_{j}(u, v)\right), \quad i \neq j,
$$

maps $D$ onto an annulus minus $n-2$ concentric circular slits with $C_{i}$ and $C_{j}$ corresponding to the two boundary circles.

From these results it is easy to obtain the desired conclusion. Fix $v_{0} \in D$ and for $i, j=0, \ldots, n-1, i \neq j$, define

$$
E_{i j}=\left\{u \in D: \lambda_{i}\left(u, v_{0}\right)=\lambda_{j}\left(u, v_{0}\right)\right\}
$$

We claim that $E_{i j}$ is nowhere dense in $D$. If, to the contrary, $\bar{E}_{i j}$ contained a nonempty open set $U$ then

$$
k_{i}\left(u, v_{0}\right)-k_{j}\left(u, v_{0}\right)=\kappa_{i}\left(u, v_{0}\right)-\kappa_{j}\left(u, v_{0}\right)
$$

would be real on $U$ and therefore reduce to a constant. In view of the mapping properties of $R_{i j}$ defined above this is clearly absurd. Finally set

$$
E=\bigcup_{i \neq j} E_{i j}, \quad i, j=0, \ldots, n-1 .
$$

Then $E$ is nowhere dense in $D$ and the lemma is proved.

Now choose $u$ and $v$ as in the lemma and let $D^{\prime}, C_{j}^{\prime}$ denote the images of $D$ and $C_{j}$ under $\Psi(z ; u, v)$. By renumbering, if necessary, we assume that $C_{0}^{\prime}, \ldots, C_{n-1}^{\prime}$ are in order of increasing argument. For each $j$ let $\xi_{j}$ be the endpoint of $C_{j}^{\prime}$ farthest from the origin and let $Q_{j}^{\prime}$ be the part of the ray containing $C_{j}^{\prime}$ that joins $\xi_{j}$ to $\infty$. The $Q_{j}^{\prime}$ meet at well determined nonzero angles at $\infty$. The rays containing the $C_{j}^{\prime}$ 
partition the plane into $n$ sectors $S_{0}^{\prime}, \ldots, S_{n-1}^{\prime}$ numbered so that $S_{j}^{\prime}$ lies between $C_{j}^{\prime}$ and $C_{j+1}^{\prime}$. (We consider all subscripts to be reduced modulo $n$.) Denote the midpoint of $C_{j}^{\prime}$ by $\omega_{j}$ and let $P_{j}^{\prime}$ be a polygonal arc joining $\omega_{j}$ to $\omega_{j+1}$ that, except for its endpoints, lies completely in $S_{j}^{\prime}$. Then

$$
P^{\prime}=\bigcup_{j=0}^{n-1} P_{j}^{\prime}
$$

is a closed polygon separating 0 from $\infty$ that does not intersect any of the $Q_{j}^{\prime}$. Let $G_{0}^{\prime}$ and $G_{1}^{\prime}$ be the components of $D^{\prime} \backslash P^{\prime}$ that contain 0 and $\infty$ respectively. Observe that for each $j, S_{j}^{\prime} \cap G_{0}^{\prime}$ is a simply-connected Jordan domain. Now define

$$
\begin{gathered}
\Delta_{0 j}^{\prime}=G_{0}^{\prime} \cup S_{j}^{\prime}, \quad \Delta_{j}^{\prime}=D^{\prime} \backslash \bar{\Delta}_{0 j}^{\prime}, \\
\mathscr{D}^{\prime}=\left\{\Delta_{0 j}^{\prime}: j=0, \ldots, n-1\right\} \cup\left\{\Delta_{j}^{\prime}: j=0, \ldots, n-1\right\} .
\end{gathered}
$$

Although the $\Delta_{j}^{\prime}, \Delta_{0 j}^{\prime}$ are not Jordan domains, they are all simply-connected. We show that $\mathscr{D}^{\prime}$ has the covering property for $D^{\prime}$ by distinguishing three possibilities for the position of two points $w_{1}, w_{2} \in D^{\prime}$. First, if $w_{1}, w_{2} \in G_{0}^{\prime}$ then $w_{1}, w_{2}$ lie in each $\Delta_{0 j}^{\prime}$. Second, if $w_{1} \in G_{0}^{\prime}$ and $w_{2} \in \bar{G}_{1}^{\prime}$ then $w_{1}, w_{2}$ lie in the closure of some $\Delta_{0 j}^{\prime}$. Finally suppose that $w_{1}, w_{2} \in \bar{G}_{1}^{\prime}$. If $w_{1}, w_{2} \in \bar{S}_{j}^{\prime}$ then $w_{1}, w_{2} \in \bar{\Delta}_{0 j}^{\prime}$. Otherwise $w_{1} \in \bar{S}_{j}^{\prime}$ and $w_{2} \in \bar{S}_{i}^{\prime}$ with $j \neq i$. Since $n \geqslant 3$ there is a $k \neq i, j$ such that $w_{1}$ and $w_{2}$ lie in the closure of $D^{\prime} \backslash \bar{\Delta}_{0 k}^{\prime}=\Delta_{k}^{\prime}$.

We will denote the various parts of $D$ corresponding under $\Psi^{-1}$ to those of $D^{\prime}$ by the same symbol without the prime. Thus we define

$$
\mathscr{D}=\left\{\Delta_{0 j}: j=0, \ldots, n-1\right\} \cup\left\{\Delta_{j}: j=0, \ldots, n-1\right\} .
$$

We claim that $\mathscr{D}$ is the required quasiconformal decomposition of $D$. In view of the above, $\mathscr{D}$ has the covering property. Under $\Psi^{-1}$ the slits open up into full circles and $G_{0}$ is a Jordan domain bounded by the piecewise analytic Jordan curve consisting of the arcs $P_{j}$ joined successively by the arcs of the $C_{j}$ lying between the two points which map to the midpoints $\omega_{j}$. (That is, joined by the arcs of the $C_{j}$ that do not contain $\Psi^{-1}\left(\xi_{j}\right)$, the preimage of the farthest endpoint.) Likewise, $S_{j}$ is bounded by the piecewise analytic curve consisting of the preimages of the rays containing $C_{j}^{\prime}$ and $C_{j+1}^{\prime}$. Since $G_{0} \cap S_{j}$ is a Jordan domain the same is true of $\Delta_{0 j}=G_{0} \cup S_{j}$. In fact $\Delta_{0 j}$ is bounded by the piecewise analytic curve consisting of $Q_{j} \cup Q_{j+1}, \cup_{i \neq j} P_{i}$ and the arcs of the $C_{i}$ that join these pieces. Furthermore, as noted earlier, at the $C_{i}$ the arcs forming $\partial \Delta_{0 j}$ meet at pairwise nonzero angles. Since all other angles are nonzero we conclude from Lemma 3 that $\partial \Delta_{0 j}$ is a quasiconformal circle for each $j$. Similar remarks apply to the domains $\Delta_{j}=D \backslash \bar{\Delta}_{0 j}$. Hence if we set

$$
K=\max \{K(\partial \Delta): \Delta \in \mathscr{D}\}<\infty
$$

then $\mathscr{D}$ is a $K$-quasiconformal decomposition of $D$. This completes the proof of Lemma 2. 
4. Other metrics and related results. If $D$ is any proper subdomain of $\mathbf{C}$ and $\varphi$ is analytic in $D$ then we define its quasihyperbolic norm to be

$$
\|\varphi\|_{D}^{*}=\sup _{z \in D}|\varphi(z)| \operatorname{dist}(z, \partial D)^{2}
$$

where $\operatorname{dist}(z, \partial D)$ is the ordinary Euclidean distance. It follows from Schwarz's lemma that

$$
\rho_{D}(z) \leqslant \operatorname{dist}(z, \partial D)^{-1}, \quad z \in D
$$

and hence

$$
\|\varphi\|_{D}^{*} \leqslant\|\varphi\|_{D}
$$

If $D$ is simply-connected then the Koebe 1/4-Theorem gives the lower bound

$$
(4 \operatorname{dist}(z, \partial D))^{-1} \leqslant \rho_{D}(z), \quad z \in D,
$$

and so for simply-connected domains we have the double inequality

$$
\|\varphi\|_{D}^{*} \leqslant\|\varphi\|_{D} \leqslant 16\|\varphi\|_{D}^{*} \text {. }
$$

Lower bounds for $\rho_{D}$ in terms of the distance to $\partial D$ in the case when $D$ is multiply-connected have been recently obtained in [4].

Once again the quasihyperbolic norm is monotonic;

$$
\|\varphi\|_{D^{\prime}}^{*} \leqslant\|\varphi\|_{D}^{*}
$$

whenever $D^{\prime} \subset D$ and $\varphi$ is analytic in $D$.

Because of (8) and (9), Theorem 3 continues to hold for the quasihyperbolic norm.

THEOREM 4. Suppose that $D$ is a finitely-connected proper subdomain of $\mathbf{C}$ and that each component of $\partial D$ is either a point or a quasiconformal circle. Then there is a positive constant $a$, depending only on $D$, such that $f$ is univalent in $D$ whenever $f$ is analytic in $D$ with $\left\|S_{f}\right\|_{D}^{*} \leqslant a$.

Proof. Let $\mathscr{D}$ be a $K$-quasiconformal decomposition of $D$. From (8) and (9)

$$
\left\|S_{f}\right\|_{\Delta} \leqslant 16\left\|S_{f}\right\|_{\Delta}^{*} \leqslant 16\left\|S_{f}\right\|_{D}^{*}
$$

for any $\Delta \in \mathscr{D}$. Now choose $a=a(K)>0$ in Theorem 2 such that $\left\|S_{f}\right\|_{\Delta}<16 a$ implies that $f \mid \Delta$ is univalent and has a quasiconformal extension to $\overline{\mathbf{C}}$. Then $\left\|S_{f}\right\|_{D}^{*} \leqslant a$ implies that $f$ is univalent in $D$ as before.

At this point we introduce domain constants associated with the two norms. (Compare [10].) If $D \subset \overline{\mathbf{C}}$ we define

$$
\begin{aligned}
& \sigma_{1}(D)=\sup \left\{\left\|S_{f}\right\|_{D}: f \text { is univalent in } D\right\} . \\
& \sigma_{2}(D)=\sup \left\{a:\left\|S_{f}\right\|_{D} \leqslant a \text { implies that } f \text { is univalent in } D\right\} .
\end{aligned}
$$

When $D \subset C$ the constants $\sigma_{1}^{*}(D)$ and $\sigma_{2}^{*}(D)$ are defined the same way using the quasihyperbolic norm. From now on whenever the quasihyperbolic norm is involved we shall assume without further comment that $\infty$ does not lie in $D$.

As an example we have that $\sigma_{1}(B)=6, \sigma_{2}(B)=2$. O. Lehto [11] has shown that if $D$ is simply-connected then $\sigma_{1}(D)<12$ and has given an example for which 
equality holds. Recently, A. F. Beardon and F. W. Gehring [3] have shown more generally that $\sigma_{1}(D)<12$ for any domain $D$. Lehto's example in the simply-connected case shows that the constant 12 cannot be improved, and Beardon and Gehring construct examples to show that 12 is also the best possible constant for each class of domains of a fixed connectivity $n=1,2,3, \ldots$

It was shown in [6] that for any domain $D, \sigma_{1}^{*}(D)<6$ and in fact that $\sigma_{2}^{*}(B)=6$. For the constant $\sigma_{2}^{*}$ we can easily show that $\sigma_{2}^{*}(B)=\frac{1}{2}$. To see this first note that

$$
\frac{1}{2} \operatorname{dist}(z, B)^{-2}=\frac{1}{2} \frac{1}{(1-|z|)^{2}}<\frac{2}{\left(1-|z|^{2}\right)^{2}}=2 \rho_{B}(z)^{2}, \quad z \in B,
$$

and, as $\sigma_{2}(B)=2$, we have $\sigma_{2}^{*}(B) \leqslant \frac{1}{2}$. Next, the function $f(z)=(1-z)^{i \alpha}$, $\alpha$ small and positive, is not univalent in $B$ and has

$$
S_{f}(z)=\frac{1+\alpha^{2}}{2} \frac{1}{(1-z)^{2}}, \quad z \in B,
$$

which shows that $\sigma_{2}^{*}(B)>\frac{1}{2}$ as well.

Finally we remark that from (6) it follows that $\sigma_{1}^{*}(D)<\sigma_{1}(D)$ and $\sigma_{2}^{*}(D)<$ $\sigma_{2}(D)$. Hence Theorem 4 is slightly stronger than Theorem 3.

The constants $\sigma_{2}$ and $\sigma_{2}^{*}$ are related to an important topological property of a domain [6].

A set $E$ in $\overline{\mathbf{C}}$ is said to be $b$-locally connected, $b \in(1, \infty)$, if for all $z_{0}$ and $r>0$, points in $E \cap \bar{B}\left(z_{0}, r\right)$ can be joined in $E \cap \bar{B}\left(z_{0}, b r\right)$ and points in $E \backslash B\left(z_{0}, r\right)$ can be joined in $E \backslash B\left(z_{0}, r / b\right)$.

Here $B\left(z_{0}, r\right)$ denotes the disc with center $z_{0}$ and radius $r$. Two points $z_{1}, z_{2} \in E$ are joined in $E$ if there is an arc $C \subset E$ having $z_{1}$ and $z_{2}$ as endpoints.

The importance of this concept for plane domains lies in the following lemma, proved in [6].

LEMMA 7. If $D$ is b-locally connected then each component of $\partial D$ is either a point or $a K$-quasiconformal circle where $K$ depends only on $b$.

The connections with the domain constants are these.

LEMMA 8 [6]. If $\sigma_{2}^{*}(D)>0$ then $D$ is b-locally connected with

$$
b \geqslant \max \left(5 / \sigma_{2}^{*}(D)+1,3\right) \text {. }
$$

LEMMA 9 [3]. If $\sigma_{2}(D)>0$ then $D$ is b-locally connected with

$$
b \geqslant \max \left(20 \pi / \sigma_{2}(D)+1,3\right) \text {. }
$$

These results allow us to deduce the following characterization.

THEOREM 5. For finitely-connected domains $D$ the following five properties are equivalent.

(i) $\sigma_{2}(D)>0$.

(ii) $D$ is b-locally connected.

(iii) $\partial D$ consists of points and quasiconformal circles.

(iv) $D$ is quasiconformally decomposable.

(v) $\sigma_{2}^{*}(D)>0$. 
Proof. Lemmas 9, 7, and 2 combine to give (i) implies (ii) implies (iii) implies (iv). That (iv) implies (v) is the proof of Theorem 4 and (v) implies (i) because $\sigma_{2}^{*}(D) \leqslant \sigma_{2}(D)$ as noted earlier.

REMARK. Lemma 9 is proved in [3] not in terms of the hyperbolic metric but in terms of the reduced Bergmann kernel function $K_{D}(z, \bar{z})$ of $D$. However, as is seen in the course of the proofs, one can just as well consider the hyperbolic metric to obtain the same conclusions. On the other hand, the sufficient conditions for univalence stated here in Theorems 2,3 , and 4 could be recast using the kernel function. Ahlfors' theorem (Theorem 2) remains true with the kernel function simply because

$$
K_{D}(z, \bar{z})=\rho_{D}(z)^{2} / \pi, \quad z \in D,
$$

when $D$ is simply-connected. The kernel function has the same monotonicity property with respect to the domain as the hyperbolic density and thus the same proof for Theorem 3 can be given using the kernel function with changes in terminology only.

We close this section by considering the connection between the constants $\sigma_{2}, \sigma_{2}^{*}$ and quasiconformal extension of conformal mappings. To do this it is necessary to use a transformation property of the two norms. If $f$ and $g$ are analytic functions then whenever their composition makes sense we have the formula

$$
S_{f \circ g}=\left(S_{f} \circ g\right)\left(g^{\prime}\right)^{2}+S_{g}
$$

In particular if $f$ is analytic in $D$ and $g$ is a conformal mapping of $D$ then we can write

$$
S_{f}-S_{g}=\left(S_{\left(f \circ g^{-1}\right)} \circ g\right)\left(g^{\prime}\right)^{2}
$$

and from the conformal invariance of the hyperbolic metric it follows that

$$
\left\|S_{f}-S_{g}\right\|_{D}=\left\|S_{f \circ g^{-1}}\right\|_{g(D)} \text {. }
$$

To determine a corresponding formula for the quasihyperbolic norm we require a simple bound for the derivative of a conformal mapping of an arbitrary domain $D \subset \mathbf{C}$. If $g$ is univalent in $D$ then

$$
\left|g^{\prime}(z)\right| \leqslant 4 \frac{\operatorname{dist}(g(z), \partial g(D))}{\operatorname{dist}(z, \partial D)}, \quad z \in D .
$$

This follows from the Koebe 1/4-Theorem by considering for each $z_{0} \in D$ and for $\zeta \in B$ the function

$$
F(\zeta)=\frac{g\left(r \zeta+z_{0}\right)-g\left(z_{0}\right)}{r g^{\prime}\left(z_{0}\right)}
$$

where $r=\operatorname{dist}\left(z_{0}, \partial D\right)$. Using this we find

$$
\left\|S_{f}-S_{g}\right\|_{D}^{*} \leqslant 16\left\|S_{f \circ g^{-1}}\right\|_{g}^{*}(D) \text {. }
$$

We can now prove an extension theorem associated with $\sigma_{2}$ and $\sigma_{2}^{*}$. 
THeOREM 6. Suppose that $D$ is a finitely-connected domain with $\sigma_{2}(D)>0$. If $f$ is analytic in $D$ with $\left\|S_{f}\right\|_{D}<\sigma_{2}(D)$ or $\left\|S_{f}\right\|_{D}^{*}<\sigma_{2}^{*}(D)$ then $f$ has a quasiconformal extension to $\overline{\mathbf{C}}$.

Proof. By Theorem 5 each component of $\partial D$ is either a point or a quasiconformal circle. From the definition, if $\left\|S_{f}\right\|_{D}<\sigma_{2}(D)$ or $\left\|S_{f}\right\|_{D}^{*}<\sigma_{2}^{*}(D)$ then $f$ is univalent in $D$ so that any point components of $\partial D$ are removable singularities. By Lemma 5 to show that $f$ has a quasiconformal extension to $\overline{\mathbf{C}}$ it suffices to show that the nondegenerate components of $\partial f(D)$ are quasiconformal circles. To do this we shall apply Theorem 5 to $f(D)$.

First assume that $\left\|S_{f}\right\|_{D}<\sigma_{2}(D)$ and suppose that $g$ is analytic in $f(D)$ with

$$
\left\|S_{g}\right\|_{f(D)}<\sigma_{2}(D)-\left\|S_{f}\right\|_{D} .
$$

Now set $h=g \circ f$. Then from (10), $\left\|S_{h}-S_{f}\right\|_{D}=\left\|S_{g}\right\|_{f(D)}$ or

$$
\left\|S_{h}\right\|_{D} \leqslant\left\|S_{f}\right\|_{D}+\left\|S_{g}\right\|_{f(D)}<\sigma_{2}(D) \text {. }
$$

Therefore $h$ is univalent in $D$ and consequently $g$ is univalent in $f(D)$. But then

$$
\sigma_{2}(f(D)) \geqslant \sigma_{2}(D)-\left\|S_{f}\right\|_{D}>0
$$

and the desired conclusion follows from Theorem 5 .

If instead we assume that $\left\|S_{f}\right\|_{D}^{*}<\sigma_{2}^{*}(D)$ then we suppose that $g$ is analytic in $f(D)$ with

$$
\left\|S_{g}\right\|_{f(D)}^{*}<\frac{1}{16}\left(\sigma_{2}^{*}(D)-\left\|S_{f}\right\|_{D}^{*}\right)
$$

Using the same argument it follows from (11) that

$$
\sigma_{2}^{*}(f(D)) \geqslant \frac{1}{16}\left(\sigma_{2}^{*}(D)-\left\|S_{f}\right\|_{D}^{*}\right)>0 .
$$

Lehto [10] proved a special case of Theorem 6 for simply-connected domains using results from the theory of Teichmüller spaces.

5. An example. In this section we give an example of an infinitely-connected domain for which both $\sigma_{2}$ and $\sigma_{2}^{*}$ are positive. As in the previous discussion this will be shown by constructing an explicit quasiconformal decomposition. In this case there will be an infinite number of elements in the decomposition and their boundary curves will consist of an infinite number of arcs, but by employing an auxiliary quasiconformal mapping we can reduce the problem of estimating the dilatations to an appeal to Lemma 3 . We begin this section by constructing this mapping and as a corollary we shall obtain the estimate in Lemma 4.

Let $C=C(\alpha)$ be the circle that intersects the unit circle $\partial B$ at the points \pm 1 at an angle $\alpha, 0<\alpha<\pi / 2$, and extends above the unit circle. We refer to $C$ as a protecting $\alpha$-circle. Let $E$ be the union of $B$ and the disc bounded by $C$ and let $B^{-}$ denote the lower half of $B$.

LEMMA 10. There is a $(1+\pi / 2 \alpha)$-quasiconformal mapping $F_{\alpha}$ of $\overline{\mathbf{C}}$ such that $F_{\alpha}(B)=B^{-}$and $F_{\alpha}$ is the identity in $\overline{\mathbf{C}} \backslash E$.

Proof. Map $B$ onto the upper half-plane by $T(z)=i(1+z) /(1-z)$. Then $C$ is mapped to a line passing through the first and third quadrants at an angle $\alpha$ while 
$B^{-}$corresponds to the first quadrant. We define a folding of the plane by

$$
f_{\alpha}\left(r e^{i \theta}\right)=f_{\alpha}(r, \theta)=\left\{\begin{array}{l}
\left(r, \frac{\theta}{2}\right), \quad 0 \leqslant \theta \leqslant \pi, \\
\left(r,\left(1+\frac{\pi}{2 \alpha}\right) \theta-\frac{\pi}{2 \alpha}(\pi+\alpha)\right), \quad \pi \leqslant \theta \leqslant \pi+\alpha, \\
(r, \theta), \quad \pi+\alpha \leqslant \theta \leqslant 2 \pi .
\end{array}\right.
$$

We also define $f_{\alpha}(0)=0, f_{\alpha}(\infty)=\infty$. Then $f_{\alpha}$ is a homeomorphism of $\overline{\mathbf{C}}$ and we easily compute the dilatation of each piece of $f_{\alpha}$ to be $2,1+\pi / 2 \alpha$, and 1 respectively. Since $0<\alpha<\pi / 2$ we have $1+\pi / 2 \alpha>2$. Since the lines $\theta=0$ and $\theta=\alpha$ are of $\sigma$-finite linear measure we conclude by a theorem of K. Strebel [15] that $f_{\alpha}$ is a $(1+\pi / 2 \alpha)$-quasiconformal mapping of $\overline{\mathbf{C}}$. The required mapping $F_{\alpha}$ is now defined by $F_{\alpha}=T^{-1} \circ f_{\alpha} \circ T$.

For use in our construction we note that any disc can be folded by conjugating $F_{\alpha}$ with a Möbius transformation.

Corollary 1. For any half-disc $D$ we have $K(\partial D) \leqslant 2$.

Proof. Without loss of generality we can take $D=B^{-}$. The estimate now follows from

$$
K\left(\partial B^{-}\right) \leqslant \inf \{1+\pi / 2 \alpha: \alpha \in(0, \pi / 2)\}=2 .
$$

Corollary 2. If $B_{1}=\{z \in B: 0<\arg (z)<4 \pi / 3\}$ then $K\left(\partial B_{1}\right) \leqslant \frac{8}{3}$.

Proof. The mapping $g(r, \theta)=(r, 3 \theta / 4)$ is a $\frac{4}{3}$-quasiconformal mapping of $\overline{\mathbf{C}}$ that maps $B_{1}$ onto the top half of $B$. The conclusion is then a consequence of Corollary 1.

For applications of a similar type of folding in three dimensions see [7].

We now construct the example. Let $\left\{B_{n}\right\}$ be a sequence of discs with $\bar{B}_{n} \subset B$, with disjoint closures and with hyperbolic centers $\left\{x_{n}\right\}, n=0,1,2, \ldots$ on the real axis. For definiteness we assume that $0=x_{0}<x_{1}<\cdots$. We further assume that the discs are chosen to have the same finite hyperbolic area and so that the hyperbolic distance between any two adjacent discs is a constant $\lambda>0$. For symmetry we set $x_{-n}=-x_{n}$ and let $B_{-n}$ be the reflection of $B_{n}$ in the imaginary axis. We define the domain $D$ to be

$$
D=B \backslash \bigcup_{n=-\infty}^{\infty} \bar{B}_{n} .
$$

A quasiconformal decomposition of $D$ is constructed as follows. The segments formed by the intersection of $D$ with the real axis join the $B_{n}$ successively and, by assumption, each has hyperbolic length $\lambda$. We let $\beta_{n}$ be the segment joining $B_{n}$ to $B_{n+1}, n=\ldots,-1,0,1, \ldots$ Next, for each $n$ let $L_{n}$ be the hyperbolic line through $x_{n}$ that is symmetric with respect to the real axis. Let $\gamma_{n}$ be the one of the two arcs formed by $D \cap L_{n}$ that lies in the lower half-plane. Let $D^{+}$and $D^{-}$be the parts of $D$ lying in the upper and lower half-planes respectively. Then the $\gamma_{n}$ partition $D^{-}$into a sequence of disjoint subdomains $D_{n}^{-}$, bounded laterally by $\gamma_{n}$ and $\gamma_{n+1}$. The domains $D^{+}$and $D_{n}^{-}$meet at the segment $\beta_{n}$ and so $\Delta_{n}=D^{+} \cup \beta_{n} \cup D_{n}^{-}$is 
again simply-connected. We now define

$$
\mathscr{D}=\left\{D^{-}\right\} \cup\left\{\Delta_{n}: n=\ldots,-1,0,1, \ldots\right\} \text {. }
$$

(D) has the covering property, for if $z_{1}, z_{2} \in D$ then there are only three possibilities to consider for their position. Either they both lie in $\bar{D}^{+}$, both lie in $\bar{D}^{-}$or one lies in $D^{+}$and one in $D^{-}$. In any of these cases both points lie in the closure of an element in $\mathscr{D}$.

The $\Delta_{n}$ are all congruent since $\Delta_{n}=T_{n}\left(\Delta_{0}\right)$ where $T_{n}$ is the Möbius transformation of the unit disc onto itself that fixes \pm 1 and maps 0 to $x_{n}$. Therefore the dilatations of their boundary curves are all the same and we need only show that $\partial \Delta_{0}$ is a quasiconformal circle. As it stands now Lemma 3 is not applicable since $\partial D^{-}$and $\partial \Delta_{0}$ consist of an infinite number of circular arcs and line segments, although they do meet at right angles by construction. However, the folding mapping of Lemma 10 will enable us to flatten the semicircles arising from the $B_{n}$ while leaving the rest of the boundary unchanged. This is so precisely because the folding was constructed to be the identity outside the disc and its protecting circle, a fact that will also allow us to fold all of the discs simultaneously.

It is necessary to provide each $B_{n}$ with a protecting $\alpha$-circle that passes through the vertices of $B_{n}$ on the real axis, extends above $B_{n}$ and lies inside $B$. Furthermore we wish to choose a single $\alpha_{0}$ so that adjacent $\alpha_{0}$-circles are at most tangent. Since the $\alpha_{0}$-circle on $B_{n}$ can be obtained from the one on $B_{0}$ by applying $T_{n}$, this will be the case if this $\alpha_{0}$-circle extends no farther to the right (and left) than the hyperbolic midpoint of $\beta_{0}\left(\beta_{-1}\right)$ and no farther above $B_{0}$ than the point $i$ on the imaginary axis. A simple calculation shows that $\alpha_{0}>0$ must be chosen so as to satisfy respectively

$$
\sec \alpha_{0} \leqslant \frac{\tanh (r+\lambda / 2)}{\tanh r}, \quad \sec \alpha_{0}+\tan \alpha_{0} \leqslant(\tanh r)^{-1}
$$

where $r$ is the hyperbolic radius of $B_{0}$. We combine these and choose $\alpha_{0}>0$ so that

$$
\sec \alpha_{0}+\tan \alpha_{0} \leqslant \frac{\tanh (r+\lambda / 2)}{\tanh r} \text {. }
$$

Let $E_{n}$ be the union of $B_{n}$ and the disc bounded by its protecting $\alpha_{0}$-circle and let $F_{n}$ be the associated folding of $B_{n}$ onto its lower half. We define a mapping $F$ of $\bar{C}$ onto itself by

$$
F(z)= \begin{cases}z, & z \in \overline{\mathbf{C}} \backslash \bigcup_{n=-\infty}^{\infty} E_{n}, \\ F_{n}(z), & z \in E_{n} .\end{cases}
$$

Because $F_{n}$ is the identity on $\overline{\mathbf{C}} \backslash E_{n}$ we see that $F$ is a homeomorphism. Moreover, for each $n,\left.F\right|_{E_{n}}$ is a $\left(1+\pi / 2 \alpha_{0}\right)$-quasiconformal mapping and because $\cup_{n=-\infty}^{\infty} \partial E_{n}$ is of $\sigma$-finite linear measure we conclude as before ([15]) that $F$ is a $\left(1+\pi / 2 \alpha_{0}\right)$-quasiconformal mapping of the entire plane.

The image of $D^{+}$under $F$ is just the upper half of the unit disc and since $K\left(\partial D^{+}\right)=K\left(\partial D^{-}\right)$it follows from Corollary 1 that $K\left(\partial D^{-}\right)<2\left(1+\pi / 2 \alpha_{0}\right)$. The image of $\Delta_{0}$ under $F$ is the mushroom shaped domain bounded by the upper 
semicircle, the real axis from -1 to 1 minus the segment $\beta_{0}$, the arcs of $\partial B_{0}, \partial B_{1}$ from the endpoints of $\beta_{0}$ to $\gamma_{0}, \gamma_{1}$ and the part of the lower half of $\partial B$ joining $\gamma_{0}$ and $\gamma_{1}$. Since all arcs meet at right angles we can now apply Lemma 3 to deduce that $\partial \Delta_{0}$ is a quasiconformal circle.

We remark that by using several more foldings it is possible to give an estimate for $K\left(\partial \Delta_{0}\right)$ but because the calculations are somewhat more involved we do not give them here. We also note that by using the same techniques it is possible to give a slightly more general example. Namely, we require that the discs $B_{n}$, still centered on the real axis, are chosen so as to satisfy the following geometric condition. Let $R_{n}$ be the ring domain bounded by $\partial B$ and $\partial B_{n}$ and for $m \neq n$ let $R_{m n}$ be the ring domain in $\overline{\mathbf{C}}$ bounded by $\partial B_{m}$ and $\partial B_{n}$. We assume that there is a positive constant $b$ such that

$$
\inf _{n} \bmod R_{n} \geqslant b, \quad \inf _{m, n} \bmod R_{m n} \geqslant b
$$

where "mod" denotes the conformal modulus.

These conditions have two consequences both of which were exemplified in the preceding construction. First, there is a finite constant $\eta=\eta(b)$ such that $\sup _{n} A\left(B_{n}\right) \leqslant \eta$ where $A\left(B_{n}\right)$ denotes the hyperbolic area of $B_{n}$ in $B$. Second, there is a positive constant $\lambda=\lambda(b)$ such that $\rho\left(B_{n}, B_{n+1}\right) \geqslant \lambda$ where $\rho\left(B_{n}, B_{n+1}\right)$ denotes the hyperbolic distance between $B_{n}$ and $B_{n+1}$. We shall not pursue this here, but we do point out that in [3] an example of an infinitely-connected domain is given for which the various univalence criteria fail by choosing the $B_{n}$ so that $\sup _{n} A\left(B_{n}\right)=$ $\infty$.

Acknowledgement. The author wishes to thank Professor F. W. Gehring for many informative conversations during the course of this work. The author would also like to record his gratitude to Professor Zeev Nehari for his interest and encouragement.

Note. The author has recently learned of a related paper, Injectivity theorems in plane and space by O. Martio and J. Sarvas to appear in Ann. Acad. Sci. Fenn. Ser. AI. The authors consider injectivity theorems related to the Schwarzian derivative but from a different point of view. They say that a domain $D$ is a uniform domain if there is a positive constant $a$ such that each pair of points $x, y \in D$ can be joined by a rectifiable curve $\gamma$ in $D$ with

$$
\min (s(x, z), s(z, y)) \leqslant a \operatorname{dist}(z, \partial D), \quad z \in \gamma,
$$

and

$$
s(x, y) \leqslant a|x-y|
$$

Here $s(x, z)$ denotes the distance along $\gamma$ from $x$ to $z$. The authors then show that $\sigma_{2}^{*}(D)>0$ for a uniform domain. This does not contain Theorem 4 of this paper since it would be necessary to establish that a domain is uniform if it is bounded by a finite number of points and quasiconformal circles. Actually, Martio and Sarvas prove a theorem in the opposite direction. 
In fact it is true that a plane domain is uniform if and only if it is quasiconformally decomposable. For this and related results we refer to a forthcoming paper, Uniform domains and the quasi-hyperbolic metric by the present author and F. W. Gehring to appear in J. Analyse Math.

\section{REFERENCES}

1. L. V. Ahlfors, Quasiconformal reflections, Acta Math. 109 (1963), 291-301.

2. L. V. Ahlfors and G. Weill, A uniqueness theorem for Beltrami equations, Proc. Amer. Math. Soc. 13 (1962), 975-978.

3. A. F. Beardon and F. W. Gehring, Schwarzian derivatives, the Poincare metric and the kernel function, Comment. Math. Helv. (to appear).

4. A. F. Beardon and $\mathrm{Ch}$. Pommerenke, The Poincaré metric of plane domains, J. London Math. Soc. (2) 18 (1978), 475-483.

5. P. R. Garabedian and M. Schiffer, Identities in the theory of conformal mappings, Trans. Amer. Math. Soc. 65 (1949), 187-238.

6. F. W. Gehring, Univalent functions and the Schwarzian derivative, Comment. Math. Helv. 52 (1977), 561-572.

7. F. W. Gehring and J. Väisälä, The coefficients of quasiconformality in space, Acta Math. 114 (1965), $1-70$.

8. E. Hille, Remarks on a paper by Z. Nehari, Bull. Amer. Math. Soc. 55 (1949), 552-553.

9. W. Kraus, Über den Zusammenhang einiger Charakteristiken eines einfach zusammenhängenden Bereiches mit der Kreisabbildung, Mitt. Math. Sem. Giessen 21 (1932).

10. O. Lehto, Domain constants associated with the Schwarzian derivative, Comment. Math. Helv. 52 (1977), pp. 603-610.

11. , Quasiconformal mappings in the plane, Lecture Notes 14, Univ. of Maryland, 1975.

12. O. Lehto and K. I. Virtanen, Quasiconformal mappings in the plane, Springer-Verlag, Berlin and New York, 1973.

13. Z. Nehari, The Schwarzian derivative and schlicht functions, Bull. Amer. Math. Soc. 55 (1949), 545-551.

14. G. Springer, Fredholm eigenvalues and quasiconformal mappings, Acta Math. 111 (1964), 121-142.

15. K. Strebel, On the maximal dilatation of quasiconformal mappings, Proc. Amer. Math. Soc. 6 (1953), 903-909.

Department of Mathematics, University of Michigan, Ann Arbor, Michigan 48109 